\title{
Design of a Supernova-relevant Rayleigh-Taylor Experiment on the National Ignition Facility. I. Planar Target Design and Diagnostics.
}

\author{
Markus Flaig $^{\mathrm{a}, *}$, Tomasz Plewa ${ }^{\mathrm{a}}$, Paul A. Keiter ${ }^{\mathrm{b}}$, R. Paul Drake ${ }^{\mathrm{b}}$, Mike Grosskopf ${ }^{\mathrm{c}}$, Carolyn Kuranz ${ }^{\mathrm{b}}$, Hye-Sook Park ${ }^{\mathrm{d}}$ \\ ${ }^{a}$ Florida State University, USA \\ ${ }^{b}$ University of Michigan, USA \\ ${ }^{c}$ Simon Fraser University, Canada \\ ${ }^{d}$ Lawrence Livermoore National Laboratory, USA
}

\begin{abstract}
We present a feasability study for a laser-driven shock experiment on the National Ignition Facility (NIF) to study the evolution of the Rayleigh-Taylor instability in the non-linear regime. The experiment is relevant to the problem of material mixing in corecollapse supernovae and is intended to serve as a stepping stone for more realistic Rayleigh-Taylor experiments using spherical geometry.

The radiation hydrodynamics simulations described here are done using the CRASH code and include the actual NIF laser drive. It is shown that the simulations are converged with respect to numerical resolution effects. Small-scale imperfections, such as they might be introduced during the process of target fabrication, are found to have negligible impact, provided that their size is smaller than $1 \mu \mathrm{m}$. The simulation results are in excellent agreement with a buoyancy-drag model, and the mix layer width is found to increase at higher drive energies.
\end{abstract}

Keywords: hydrodynamics, hydrodynamic instabilities, laboratory astrophysics, Rayleigh-Taylor, HEDP laboratory experiments, National Ignition Facility

\section{Introduction}

Astrophysical observations (particularly of SN 1987A) suggest that during a core-collapse supernova, extensive mixing occurs between the material shells that make up the onion-like structure of the exploding star. It is believed that the mixing is caused mainly by two types of fluid instabilities, namely the Richtmeyer-Meshkov instability (RMI, [27, 33]), which occurs when the supernova shock crosses material interfaces, and the Rayleigh-Taylor instability (RTI, [32, 36]), which develops as a consequence of the deceleration of the supernova shock as it passes through the progenitor envelope. The mixing might also be increased by the Kelvin-Helmholtz instability or the onset of turbulence.

Already shortly after the discovery of SN 1987A, multidimensional numerical simulations were employed to help understand the observed mixing [1, 9, 17, 18, 19]. However, due to the approximate nature of computational models and limited computer resources, such calculations are limited in several respects. In general, the computer models were not successfull in reproducing either the observed velocities of the heavy elements that are mixed from the stellar core to the envelope during the explosion [10] or the amount of hydrogen mixed into the central regions of the ejecta [14]. Both properties are required for the models to explain observations.

${ }^{*}$ Corresponding Author.

E-mail address: mflaig@fsu.edu (M. Flaig)
Better understanding of the mixing taking place in corecollapse supernovae can be obtained from high-energy density laboratory experiments $[5,23,22]$. In this approach, one uses similarity scaling [35] to reproduce the physical conditions prevailing in the envelope of the exploding star during the shock passage. The similarity scaling holds as long as viscous and radiative effects (i.e. thermal conduction and radiative transport) can be neglected. In addition, since the mixing in supernovae occurs at very high Reynolds number and may involve turbulence, the Reynolds number in the experiments has to be greater than the threshold above which the transition to turbulence occurs [29].

A series of laboratory experiments which exploited the aforementioned scaling analysis by [35, Ryutov] was executed on the Nova laser $[16,15]$. These experiments were guided by computational studies of supernova explosions with the material interface velocity, density and pressure gradients matching the behaviour observed in such simulations. They used planar target geometry with two- and three-dimensional perturbations, and demonstrated that the RTI grows significantly faster in the case when perturbations are three-dimensional. Drake et al. [6] reported an attempt to extend this approach to hemispherical target geometry on the Omega laser, and found that much higher laser energy would be necessary to successfully execute such experiments. Robey et al. [34] performed supernova RTI experiments on the Omega laser with planar targets using twodimensional single- and multimode perturbations. The results of those experiments were analyzed by Miles et al. [29], who 
found good agreement between a buoyancy-drag model [25, 11] and the single mode experiment, while the agreement was poor in the case of multimode perturbations. Later work by Kuranz et al. [22, 20, 21] continued on the Omega laser using planar targets, again finding good agreement with the predictions of a buoyancy-drag model. The experimental radiographic images indicated the presence of plasma protruding from the tips of the RTI spikes toward the shock front. Subsequent numerical simulations consistently failed to reproduce these features [22, 30].

In this work, we present and discuss the results of design simulations of a Rayleigh-Taylor experiment tailored to the $\mathrm{Na}$ tional Ignition Facility (for another recently proposed directdrive experiment, see [26]). In this experiment, the lasers would directly strike and ablate a low-Z material in planar geometry. Since no experiment of this type has yet been executed on NIF, performing such an experiment would be an important step toward developing a planar, directly-driven platform and the associated diagnostics on NIF. In addition, such an experiment would also constitute a stepping stone for more realistic designs using hemispherical targets (see, for example, [12, 13]). Among the available experimental facilities, such experiments can currently only be executed on NIF.

The NIF laser includes 192 beams arranged on two sides, in opposing sets of 96 beams. Within each set of 96 beams, the beams are conically arranged at angles 23, 30, 44.5 and 50 degrees. The target is directly driven by a subset of beams from one side of NIF. Thermal conduction, viscosity and magnetic fields are neglected in the simulations, while radiative transport is included. The focus is on general design parameters such as the target dimensions, the influence of target fabrication imperfections, the dependence of the flow morphology on the drive energy, effects due to numerical diffusion and the performance of the diagnostics systems.

The outline of the paper is as follows: In Sec. 2, we describe the computational model used to simulate the proposed experiment. In Sec. 3, the base design model is presented. In Sec. 4 we discuss the dependence of the designed experiment outcomes on numerical model resolution, small scale perturbations on the target, and the laser drive energy. Synthetic radiographs are calculated from the simulation data in order to demonstrate the diagnosability of the experiment. We also compare the growth of the mix layer width to predictions from a buoancy-drag model. In Sec. 5, we summarize our findings and give an outlook of the future work planned.

\section{Model}

\subsection{General approach}

The experiment we have in mind uses 64 laser beams to drive a shock wave down the axis of a cylindrical tube, which contains multiple layers of interest, described in Sec. 2.2. Additional beams are tasked with generating $\mathrm{x}$-ray photons which are used to diagnose the material mixing that develops as a consequence of RMI and RTI.

We perform two-dimensional numerical simulations of the proposed experiment using the CRASH code [37], which solves

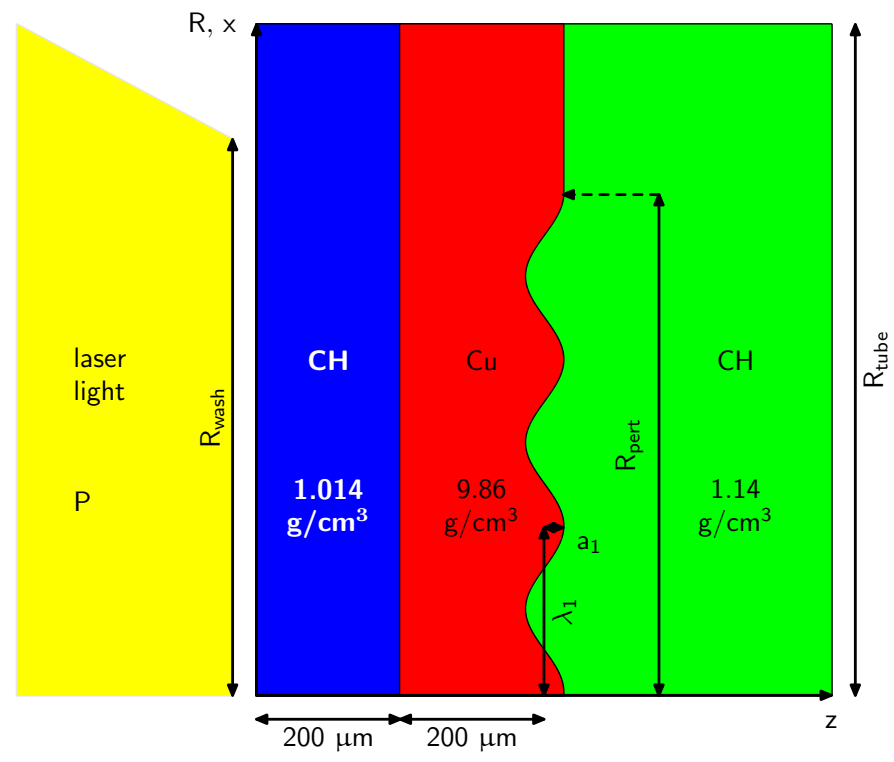

Figure 1: Simulation setup. Simulations are carried out either in Cartesian $(x, z)$ or cylindrical $(R, z)$ geometry. The target consists of a $200 \mu \mathrm{m}$ thick ablator, followed by a $200 \mu \mathrm{m}$ copper layer and a bromium-doped plastic layer. The target radius is denoted by $R_{\text {tube }}$. The perturbation on the $\mathrm{Cu} / \mathrm{CH}$ interface is characterized by wavelength $\lambda_{1}$ and amplitude $a_{1}$ and is confined to the region inside the radius $R_{\text {pert }}$. In addition to this primary perturbation, some of the simulations have an additional, small-scale perturbation with wavelenght $\lambda_{2}$ and amplitude $a_{2}$ on one or more of the interfaces imposed (not shown). $P$ denotes the total power of the incoming laser beams. The laser heating is restricted to the region with distance $\leq R_{\text {wash }}$ from the $z$ axis.

the equations of radiation hydrodynamics on dynamically adaptive Eulerian meshes. CRASH includes flux-limited multigroup radiation diffusion, thermal heat conduction, multi-material treatment (using the level set method) and the capability to calculate the energy deposition due to the illumination by the laser in the geometrical optics approximation [38]. The simulations presented here include both radiative transport and thermal conduction. From the set of availabe Riemann solvers, we choose the Linde flux. SESAME tables are used for the calculation of the equation of state. The mesh is adaptively refined during the simulation in regions with large density variations and at material interfaces. The initial energy deposition due to the laser illumination is calculated with parameters corresponding to the actual NIF laser drive configuration (Sec. 2.3).

Based on the simulation results, we calculate simulated radiographs in order to demonstrate the diagnosability of the experiment and to optimize the experiment design. The method used to calculate the radiographs (Sec. 2.4) combines the data from a 2D Cartesian simulation (valid in the region near the tube axis) and a 2D cylindrical simulation (valid outside the perturbed region).

\subsection{Basic setup}

Fig. 1 contains a sketch of the setup used in the numerical simulations. The target is assumed to be a cylindrical tube consisting of a $200 \mu \mathrm{m}$ thick plastic ablator with density $1.044 \mathrm{~g} / \mathrm{cm}^{3}$, which is followed by a $200 \mu \mathrm{m}$ thick copper layer with density $8.96 \mathrm{~g} / \mathrm{cm}^{3}$ and a plastic layer with density 
$1.14 \mathrm{~g} / \mathrm{cm}^{3}$. The target radius is denoted by $R_{\text {tube }}$, which is set to $R_{\text {tube }}=1400 \mu \mathrm{m}$ for most of the simulations.

The value of $1.14 \mathrm{~g} / \mathrm{cm}^{3}$ for the density of the final plastic layer corresponds to $1 \%$ brominated plastic which would be advantageous for the diagnostics. The density of the vacuum around the target is set to a small value $\left(10^{-5} \mathrm{~g} / \mathrm{cm}^{3}\right)$ in order to prevent the occurence of zero densities in the numerical scheme. The material in the vacuum region is set to beryllium.

The target has a sinusoidal perturbation imposed on the $\mathrm{Cu} / \mathrm{CH}$ interface, characterized by wavelength $\lambda_{1}$ and amplitude $a_{1}$ (Fig. 1). The perturbation is restricted to the region inside the "perturbation radius" $R_{\text {pert }}$, with the interface being smooth outside this region. We choose $R_{\text {pert }}=750 \mu \mathrm{m}$ for all simulations presented in this paper. Some of the simulation have an additional perturbation with wavelength $\lambda_{2}$ and amplitude $a_{2}$ on one or more of the interfaces (see Sec. 4.2).

The simulations are carried out either in cylindrical $(R, z)$ or Cartesian $(x, z)$ geometry, with the $z$ axis in both cases corresponding to the longitudinal direction (along the tube axis) and the $R(x)$ axis corresponding to the lateral direction (perpendicular to the tube axis). The longitudinal domain size is $L_{z}=2560 \mu \mathrm{m}$ for all simulations. The lateral domain size $L_{R}\left(L_{x}\right)$ is either equal to the longitudinal domain size ("fulldomain" simulations) or half the perturbation wavelength $\lambda_{1}$ ("small-domain" simulations). All simulations employ reflecting boundary conditions at the lower $R(x)$ boundary and outflow boundary conditions at the left and right $z$ boundaries. The boundary conditions at the upper $R(x)$ boundary are outflow for the full-domain simulations and reflecting for the small-domain simulations.

\subsection{Laser drive configuration}

The ablator surface is illuminated using the $23^{\circ}, 30^{\circ}$ and $50^{\circ}$ beams of the NIF laser, while the $44.5^{\circ}$ beams are reserved for the diagnostics (see [7] for information on the beam configuration on NIF). The NIF laser beams are of elliptical Gaussian shape, which we approximate by circular beams having the same area. The $23^{\circ}$ and $30^{\circ}$ beams point to the center of the target while the $50^{\circ}$ beams are offset from the center by a distance $R_{\text {off }}$ (see Fig. 2 for a sketch of the laser drive configuration). In the numerical simulations, the beams do not interact, i.e. there is no cross-beam energy transfer, which in an actual experiment might affect the irradiation patterns [8]. The pulse shape that is assumed in the simulations consists of a linear raise lasting $125 \mathrm{ps}$, followed by constant power for $3 \mathrm{~ns}$ and a linear decay lasting another $125 \mathrm{ps}$.

We denote the combined power carried by the $23^{\circ}$ and $30^{\circ}$ beams by $P_{\text {ctr }}$. In order to achieve approximately uniform laser illumination in the perturbed region, we vary $R_{\mathrm{off}}$ and $P_{\mathrm{ctr}} / P$ such that the intensity variations in this region are minimized, i.e. we minimize

$$
\delta I^{2}=\left\langle(I-\langle I\rangle)^{2}\right\rangle,
$$

where $I$ denotes the laser intensity on the ablator surface and $\langle\ldots\rangle$ is the arithmetic mean calculated over the region $R<R_{\text {pert }}$.

In the upper panel of Fig. 3, we plot the intensity variations $\delta I /\langle I\rangle$ vs. the offset $R_{\text {off }}$ for different values of $R_{\text {pert }}$. For the

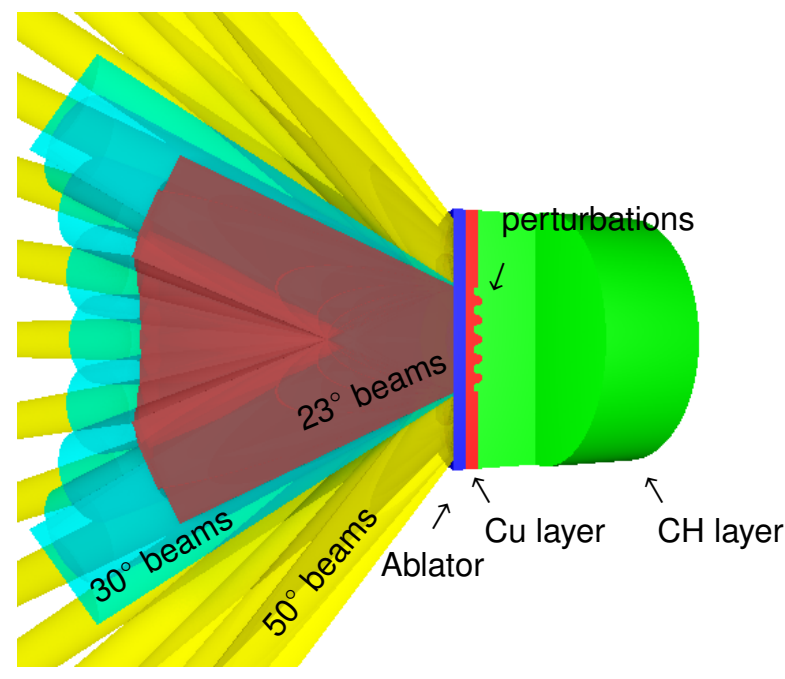

Figure 2: Sketch of the laser drive configuration used in the numerical simulations. The $23^{\circ}$ (brown) and $30^{\circ}$ (blue) beam bundles are comprised of 16 beams each; the beams point toward the center and carry $20 \%$ of the total laser energy. The $50^{\circ}$ (yellow) beam bundle consists of 32 beams that are offset from the center by a distance $R_{\text {off }}=1100 \mu \mathrm{m}$ and that carry $80 \%$ of the total energy. The target radius in this sketch is $2000 \mu \mathrm{m}$.
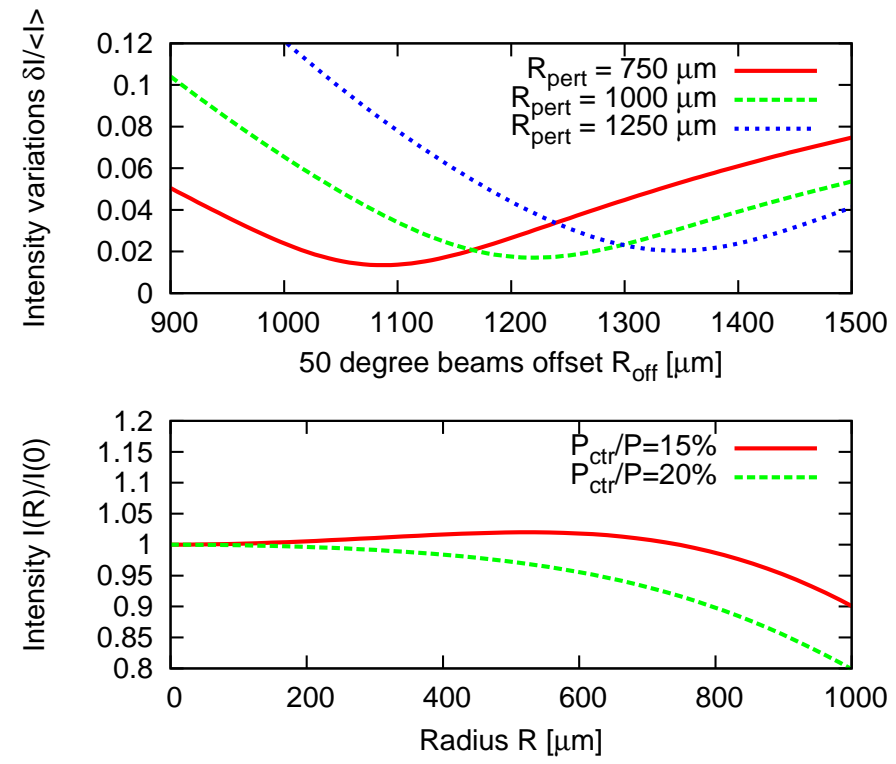

Figure 3: In the upper panel, the r.m.s. variations in the laser intensity in the region $R<R_{\text {pert }}$ are plotted vs. the the $50^{\circ}$ beams offset for different values of $R_{\text {pert }}$. For the value of $R_{\text {pert }}=750 \mu \mathrm{m}$ that is used in the simulations, the optimal offset is $R_{\text {off }} \approx 1100 \mu \mathrm{m}$. Choosing this offset, the lower panel shows the intensity profiles for the cases where $P_{\mathrm{ctr}} / P=0.15$ (optimal in terms of the uniformity of the illumination) and $P_{\text {ctr }} / P=0.20$ (optimal in terms of shock wave planarity). 
case $R_{\text {pert }}=750 \mu \mathrm{m}$, we obtain optimal values $R_{\text {off }} \approx 1100 \mu \mathrm{m}$ and $P_{\mathrm{ctr}} / P \approx 0.15$. The corresponding lateral profile of the intensity is plotted in the lower panel of Fig. 3 (the red curve), showing that the intensity variations are smaller than $10 \%$ out to $R=1000 \mu \mathrm{m}$. For the numerical simulations, we adopt $R_{\text {off }}=1100 \mu \mathrm{m}$ and $P_{\text {ctr }} / P=0.2$, since we find that choosing this somewhat higher value for $P_{\text {ctr }} / P$ leads to even better results in terms of the planarity of the shock wave, although the irradiation profile is then less uniform (Fig. 3). This is not unexpected, since the target expansion will inevitably influence the shock wave profile.

The laser illumination is assumed to be limited to a region with distance $<R_{\text {wash }}$ from the tube axis, as sketched in Fig. 1 . In an experiment, this would be achieved by means of a socalled "washer", a disc of high-Z material with a hole in the center that is attached to the ablator surface in order to shield the region outside of the hole from the laser illumination. In the simulations, this effect is accounted for by setting the laser heating to zero outside the radius $R_{\text {wash }}$. For most of the simulations presented in this paper, we set $R_{\text {wash }}=1000 \mu \mathrm{m}$.

To summarize, the canonical parameters that are used in most of the simulations, are $R_{\text {tube }}=1400 \mu \mathrm{m}$ for the tube radius, $R_{\text {pert }}=750 \mu \mathrm{m}$ for the "perturbation radius", $R_{\text {off }}=1100 \mu \mathrm{m}$ for the $50^{\circ}$ beams offset, $P_{\mathrm{ctr}} / P=0.2$ for the ratio of laser power in the centered vs. the off-centered beams and $R_{\text {wash }}=$ $1000 \mu \mathrm{m}$ for the laser cut-off. Table 1 provides an overview on all simulations presented in this paper.

\subsection{Diagnostic model}

We assume that the experiment is diagnosed via X-ray radiography [24]. For the calculation of synthetic radiographs, we combine the opacity $\kappa_{\text {cart }}(x, z)$ from a 2D Cartesian simulation and the opacity $\kappa_{\text {cyl }}(R, z)$ from a $2 \mathrm{D}$ cylindrical simulation into three-dimensional dataset $\kappa(x, y, z)$ from which the radiographs are calculated. Apart from the geometry, the cylindrical simulation has the same parameters as the Cartesian one. The data are combined such that inside a certain radius (labeled $R_{\text {cart }}$ ) the data from the Cartesian simulation is used, while outside the data from the cylindrical simulation is used; i.e. we set

$$
\kappa(x, y, z)=\left\{\begin{array}{ll}
\kappa_{\mathrm{cart}}(x, z) & ; R<R_{\mathrm{cart}} \\
\kappa_{\mathrm{cyl}}(R, z) & ; R>R_{\mathrm{cart}}
\end{array},\right.
$$

where $R=\sqrt{x^{2}+y^{2}}$.

The rationale behind Eq. (2) is that in the case where the target has a non-zero planar perturbation on the $\mathrm{Cu} / \mathrm{CH}$ interface, then the azimuthal symmetry is broken in the perturbed region and data from a cylindrical simulation will be a good representation of the physical reality only outside of the perturbed region. A Cartesian simulation, on the other hand, does not represent the region far away from the tube axis well, but is always applicable to the region near the tube axis, even if that region is perturbed.

In order to guide our choice of $R_{\text {cart }}$, we demand that in the unperturbed cylindrical simulation, the lateral variations of the mean free path of the $\mathrm{x}$-ray photons be small in the region where

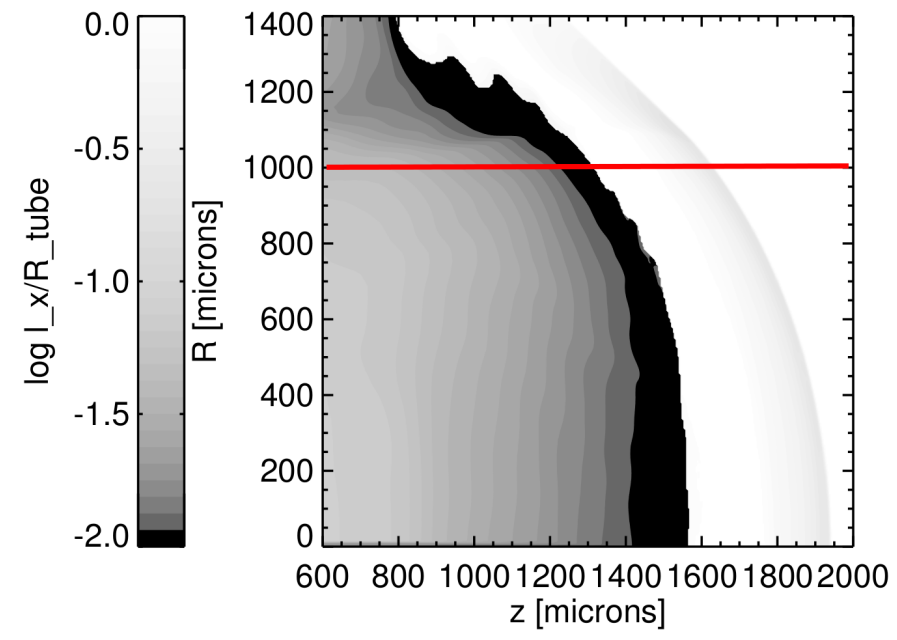

Figure 4: Plot showing the mean free path $\ell_{\mathrm{x}}$ of the $\mathrm{x}$-ray photons for the simulation U300T1400, at time $60 \mathrm{~ns}$. The opacity used to calculate the optical depth corresponds to $\mathrm{x}$-ray photons generated by a zinc backlighter. The horizontal line corresponds to $R_{\text {cart }}=1000 \mu \mathrm{m}$.

$R<R_{\text {cart }}$. In Fig. 4, we plot the mean free path of the x-ray photons for the base simulation presented in Sec. 3. In the region where the material mixing takes place, the lateral variations are mostly below $10 \%$ for $R<1000 \mu \mathrm{m}$. For the remainder of the paper, we adopt $R_{\text {cart }}=1000 \mu \mathrm{m}$.

For the calculation of the simulated radiographs, we assume parallel rays, which is justified by the fact that the distance of the backlighter to the target is significantly greater than the target size. For all radiographs shown in the paper, the backlighter material is assumed to be zinc. From the possible noise sources, we include only photon statistical noise, since at the low photon counts in the region of interest (see Sec. 3), this will be the dominant noise source. We do not include motion blur (smearing of the radiographs due to the plasma motion), because the contribution is smaller than the pinhole resolution.

\subsection{Unperturbed simulations}

We perform a series of low-resolution simulations of an unperturbed target with different target diameters and laser energies. These simulations serve mainly to justify our choice for the target radius $R_{\text {tube }}=1400 \mu \mathrm{m}$. The simulations are carried out in cylindrical geometry. The simulation parameters can be inferred from Table 1 (first four rows, simulation labels starting with 'U').

The incident laser pulse drives a shock wave into the target. When the laser drive ends after $3.25 \mathrm{~ns}$, a rarefaction wave is launched which eventually catches up with the shock and turns it into a blast wave. The blast wave passes the $\mathrm{Cu} / \mathrm{CH}$ interface at $\sim 12 \mathrm{~ns}$, and at later times the interface experiences deceleration until the end of the simulation.

Fig. 5 shows the signal-to-noise (SNR) ratio of the simulated radiographs for these simulations. The SNR is averaged over the region defined by $1000 \mu \mathrm{m}<z<2200 \mu \mathrm{m}$ and $R<$ $1500 \mu \mathrm{m}$, which, at the time where the radiographs are taken, contains the mixing region as well as the shock front. The plots show that the choice $R_{\text {tube }}=1400 \mu \mathrm{m}$ is indeed a good choice 


\begin{tabular}{lllllllll}
\hline \hline Model & $E / \mathrm{kJ}$ & $L_{x} / \mu \mathrm{m}$ & $R_{\text {tube }} / \mu \mathrm{m}$ & $\lambda_{1} / \mu \mathrm{m}$ & $a_{1} / \mu \mathrm{m}$ & $\lambda_{2} / \mu \mathrm{m}$ & $a_{2} / \mu \mathrm{m}$ & $\lambda_{1} / \Delta x_{\min }$ \\
\hline U100T $* \mathrm{X}$ & 100 & 2560 & $*=1000,1100, \ldots, 2000$ & 250 & 0 & - & - & 64 \\
U100T $*$ & 100 & 2560 & $*=1000,1100, \ldots, 2000$ & 250 & 0 & - & - & 64 \\
U200T $*$ & 200 & 2560 & $*=1000,1100, \ldots, 2000$ & 250 & 0 & - & - & 64 \\
U300T $*$ & 300 & 2560 & $*=1000,1100, \ldots, 2000$ & 250 & 0 & - & - & 64 \\
P100L128 & 100 & 2560 & 1400 & 250 & 15 & - & - & 128 \\
P200L128 & 200 & 2560 & 1400 & 250 & 15 & - & - & 128 \\
P300L128 & 300 & 2560 & 1400 & 250 & 15 & - & - & 128 \\
P300L256 & 300 & 2560 & 1400 & 250 & 15 & - & - & 256 \\
SL $*$ & 80 & 128 & - & 256 & 24 & - & - & $* 32,64, \ldots, 2048$ \\
SVA $(*, \dagger)$ & 80 & 128 & - & 256 & 24 & $*=26,52,108$ & $\dagger=1, \ldots, 8$ & 1024 \\
SAC $(*, \dagger)$ & 80 & 128 & - & 256 & 24 & $*=26,52,108$ & $\dagger=1, \ldots, 8$ & 1024 \\
SCP $(*, \dagger)$ & 80 & 128 & - & 256 & 24 & $*=26,52,108$ & $\dagger=1, \ldots, 8$ & 1024 \\
SVAHR & 80 & 128 & - & 256 & 24 & 52 & 0.5 & 2048 \\
SCPHR & 80 & 128 & - & 256 & 24 & 52 & 0.5 & 2048 \\
SW & 80 & 128 & - & 256 & 24 & 52 & 1 & 1024 \\
\hline
\end{tabular}

Table 1: Simulation overview. Simulation names (first column) starting with 'U' ("unperturbed") correspond to full-domain simulations without any perturbations, carried out in cylindrical geometry. Names starting with 'P' ("perturbed") correspond to full-domain Cartesian simulations with a perturbation on the Cu/CH interface. An 'S' denotes small-domain simulations with lateral domain size $L_{x}=\lambda_{1} / 2$, which are also done Cartesian geometry. The laser energy (second column) is stated as the total energy carried by all laser beams. The third column states the lateral extension of the computational domain (the longitudinal extent being $L_{z}=2560 \mu \mathrm{m}$ for all simulations). Column no. 4 states the target radius. In the fifth and sixth column, the wavelength and the amplitude of the primary perturbation on the $\mathrm{Cu} / \mathrm{CH}$ interface are given. The wavelength and amplitude of the small-scale secondary perturbation are listed in the seventh and eigth column. For the simulations starting with 'SVA', the secondary perturbation is located on the ablator surface, while for those starting with 'SAC' ('RCP'), it is located on the ablator/copper (copper/plastic) interface. The last column lists the effective resolution in terms of the number of grid cells that fit inside the wavelength $\lambda_{1}$. Simulation 'SW' ("worst case") has secondary perturbations with equal wavelength, amplitude and phase on the ablator surface, the ablator/copper and the copper/plastic interface simultaneously.

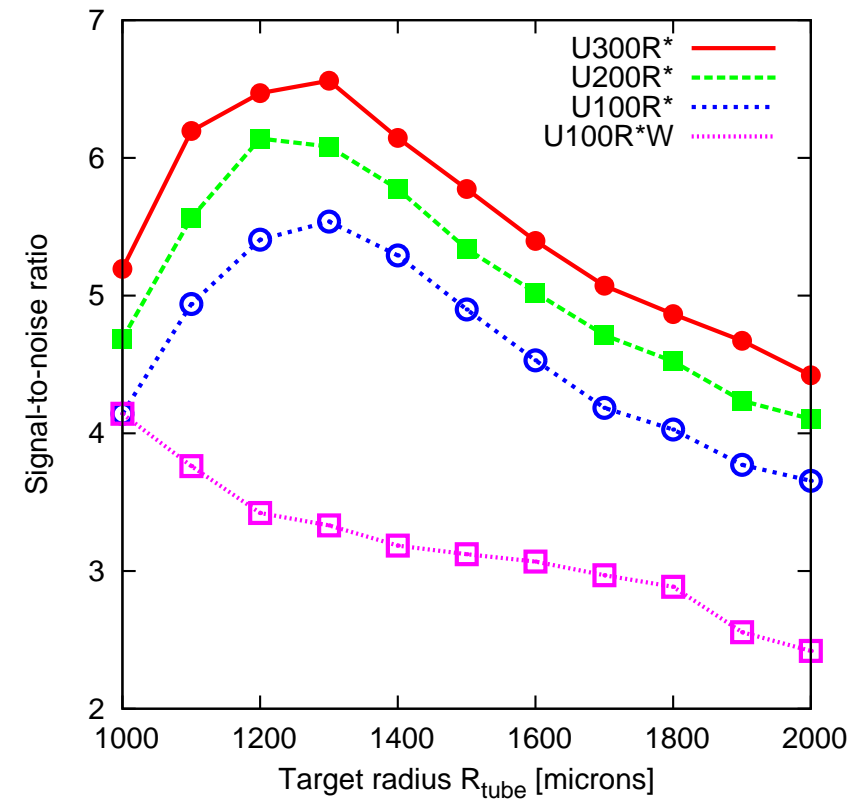

Figure 5: Signal-to-noise ratio (SNR) vs. target radius for different drive energies. The SNR is defined as $1 / \sqrt{N}$, where $N$ is the number of photons per resolution element. The backlighter material is assumed to be zinc and the pinhole size is $25 \mu \mathrm{m}$. The simulated radiographs from which the SNR was calculated were taken at time $t=140 \mathrm{~ns}$ for the $100 \mathrm{~kJ}$ cases, at $t=94 \mathrm{~ns}$ for the $200 \mathrm{~kJ}$ case and at $t=70 \mathrm{~ns}$ for the $300 \mathrm{~kJ}$ case. All simulations use the standard laser cut-off $R_{\text {wash }}=1000 \mu \mathrm{m}$, except for the simulations from the $\mathrm{U} 100 \mathrm{~T} * \mathrm{X}$ series, where the cut-off is set equal to the target radius. for the laser energies considered. Fig. 5 also includes the SNR from a series of $100 \mathrm{~kJ}$ simulations (U100T*X series), where the cut-off for the laser illumination has been set to $R_{\text {wash }}=R_{\text {tube }}$ instead of using the standard value $R_{\text {wash }}=1000 \mu \mathrm{m}$. For these simulations, the SNR is generally much smaller, which demonstrates the importance of using the cut-off for the laser illumination.

In Fig. 6, we show sample radiographs for some of the simulations appearing in Fig. 5. In the simulation shown in the top left panel, where $R_{\text {tube }}=1400 \mu \mathrm{m}$ and the whole ablator surface is illuminated, the field of view is polluted by a cloud of copper (marked by an arrow). As a consequence, the SNR is much lower than in the case where the laser illumination is cut off at the fixed radius $R_{\text {wash }}=1000 \mu \mathrm{m}$ (bottom left panel). This overshooting of copper into the field of view is also the reason for the non-monotonic behaviour of the SNR vs. target radius curves in Fig. 5 for the simulations with fixed laser cutoff. The simulations shown in the right panels in Fig. 5 have identical parameters as those in the left left panels, except that the target radius is bigger $\left(R_{\text {tube }}=2000 \mu \mathrm{m}\right.$. The contrast ist again much better in the case with fixed laser cut-off. In addition to this, Fig. 6 also reveals that the planarity of the interface is much better preserved in the simulations using the fixed laser cut-off.

\section{Results}

We choose simulation P300L256 (see Table 1) as the reference model, having a target radius of $1400 \mu \mathrm{m}$ and a laser drive energy of $300 \mathrm{~kJ}$. The specific intensity in the region 

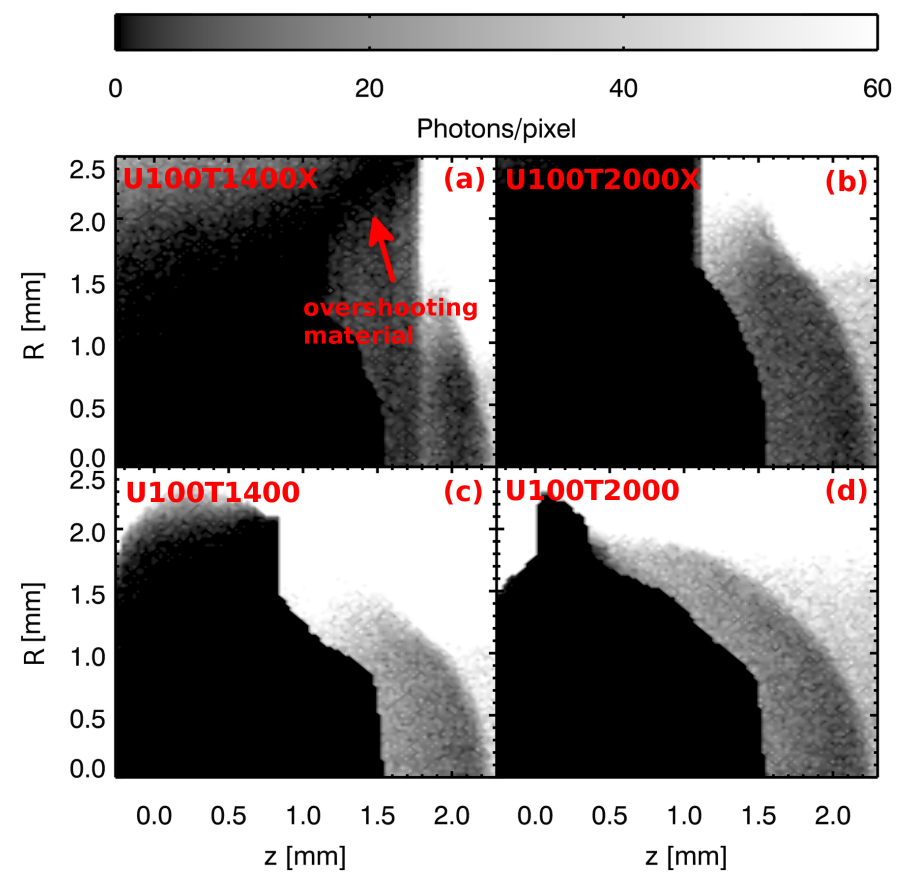

Figure 6: Synthetic radiographs at time $t=140 \mathrm{~ns}$ for the case of an unperturbed interface and $100 \mathrm{~kJ}$ drive energy. For the simulations of the top panel, the target is illuminated out to the target boundary $\left(R_{\text {wash }}=R_{\text {tube }}\right)$, while for the simulations of the bottom panel, the laser illumination has been cut off at $R_{\text {wash }}=1000 \mu \mathrm{m}$. The left plot in each panel corresponds to a target radius $R_{\text {target }}=1400 \mu \mathrm{m}$, while the right plot has $R_{\text {target }}=2000 \mu \mathrm{m}$. In the top left panel, an arrow marks material that is overshooting into the forward direction. For an interpretation of the radiographs, see the text.

$R<1000 \mu \mathrm{m}$ is $\sim 1.3 \times 10^{15} \mathrm{~W} / \mathrm{cm}^{2}$. Choosing a high drive energy has the advantages of leading to faster instability growth, thereby reducing the time delay needed between the drive and diagnostic beams, and also yielding better contrast in the radiographs (see Sec. 4.3 below). The perturbation on the $\mathrm{Cu} / \mathrm{CH}$ interface has wavelength $\lambda_{1}=250 \mu \mathrm{m}$ and amplitude $a_{1}=15 \mu \mathrm{m}$. The effective resolution is $1 \mu \mathrm{m}$, corresponding to 250 resolution elements per wavelength. The simulation is performed in Cartesian geometry and the domain size is $2560 \mu \mathrm{m}$ in both directions.

The initial density distribution is shown in Fig. 7 (a). The target is embedded in a relatively large low-density region representing the vacuum. The perturbed section of the interface is located at $x=400 \mu \mathrm{m}$ and extends vertically to $750 \mu \mathrm{m}$; the remaining part of the interface is unperturbed. The shock crosses the interface at $t \approx 9 \mathrm{ns,}$ as shown in Fig. 7 (b). At this time, the central portion of the shock front appears approximately flat, as desired. The density changes visible along the central portion of the shock front are due to the perturbations imposed on the interface. The curving of the shock at large distances from the center of the target is due to a rarefaction wave that enters the target at the target-vacuum boundary and moves towards the target's center. The associated large lateral pressure gradient can be clearly seen in Fig. 8 (b), where the pressure at $t=60 \mathrm{~ns}$ is plotted. At later times, panels (c) and (d) in Fig. 7, the curvature of the shock front becomes more pronounced, but the curvature of the $\mathrm{Cu} / \mathrm{CH}$ interface stays small in the central part of the tar-
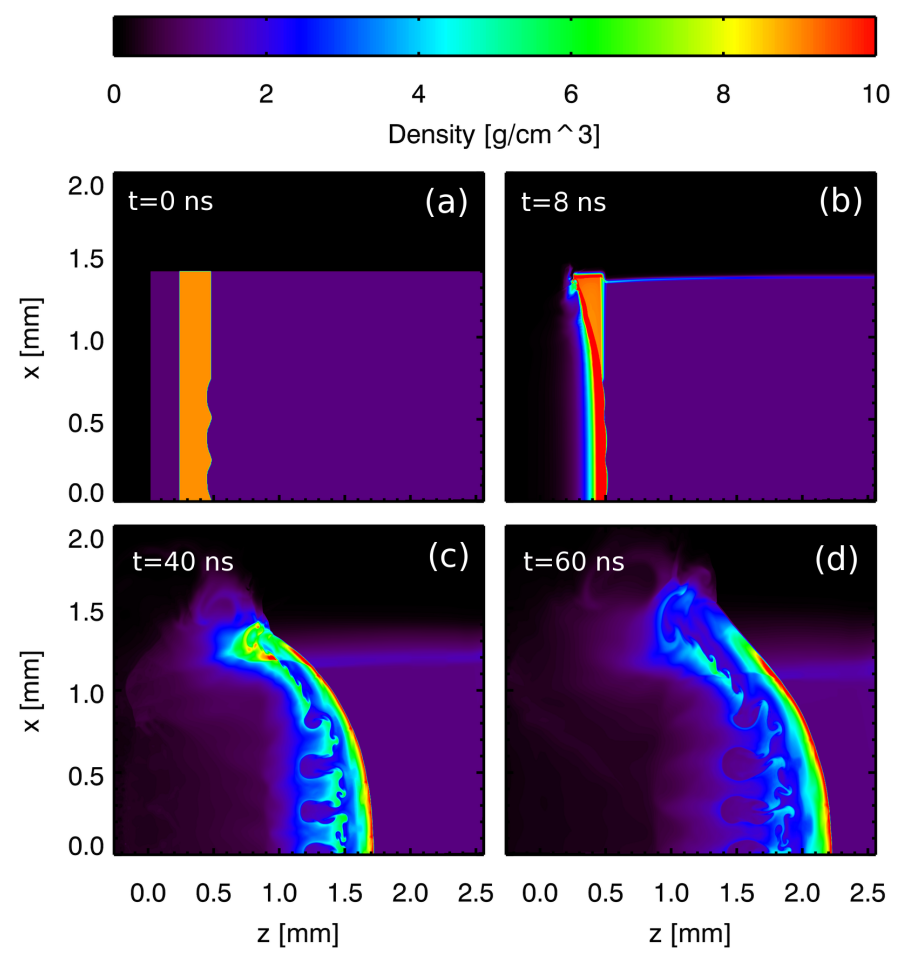

Figure 7: Density plots from the reference model at different times. Panel (a) shows the initial state at $t=0$. The state at the time when the shock crosses the $\mathrm{Cu} / \mathrm{CH}$ interface ( $t=8 \mathrm{~ns}$ ) is plotted in panel (b). Panels (c) and (d), correspondig to times $t=40 \mathrm{~ns}$ and $t=60 \mathrm{~ns}$, respectively, show the development of the Rayleigh-Taylor instability as the interface is decelerated.
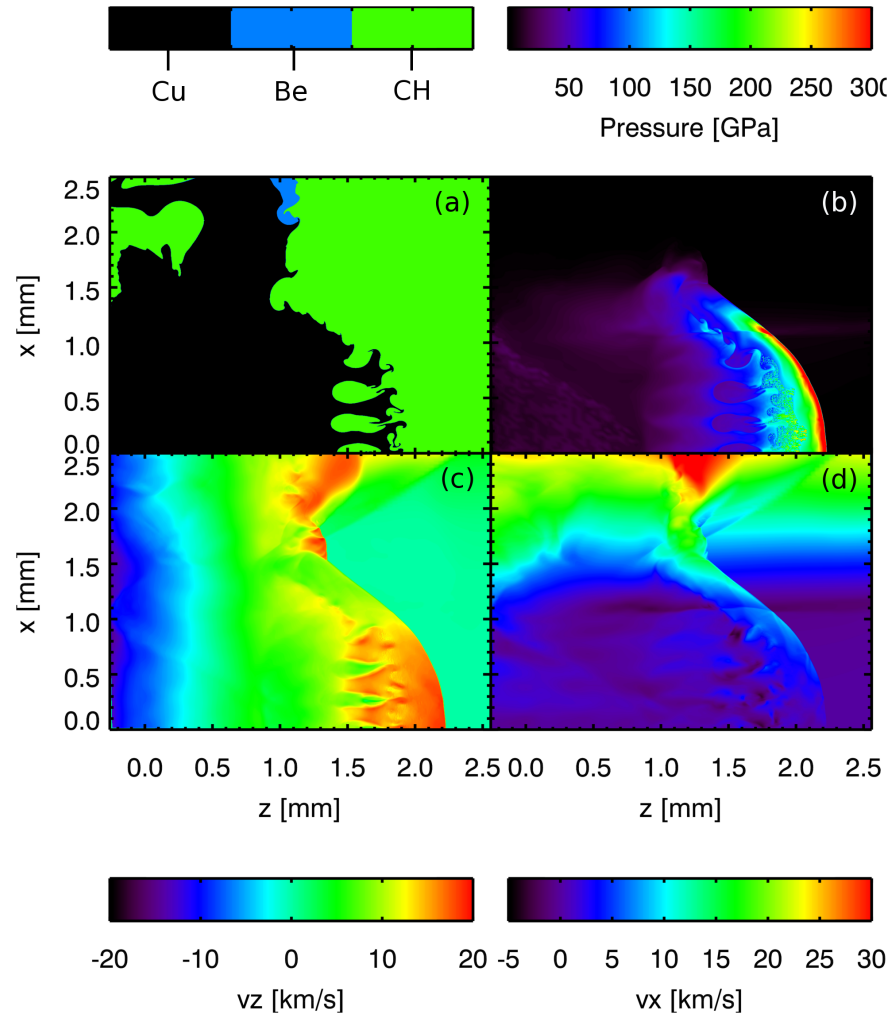

Figure 8: Plots showing the final state of the reference model at $60 \mathrm{~ns}$. Panel (a) shows the materials, panel (b) depicts the pressure, and panels (c) and (d) show the longitudinal and lateral velocities, respectively. For a discussion of the features in these plots, see the text. 
get. During the course of the simulation, the Atwood number drops from its initial value of $A=0.77 \mathrm{~g} / \mathrm{cm}^{3}$ to $A \sim 0.3 \mathrm{~g} / \mathrm{cm}^{3}$ at $t=40 \mathrm{~ns}$ and reaches a value of $A \sim 0.2 \mathrm{~g} / \mathrm{cm}^{3}$ at $t=60 \mathrm{~ns}$.

The Rayleigh-Taylor instability produces three well resolved spikes, and the mix layer width increases from slightly less than $300 \mu \mathrm{m}$ at $40 \mathrm{~ns}$ to about $500 \mu \mathrm{m}$ at $60 \mathrm{~ns}$. The relative motion of Rayleigh-Taylor fingers and bubbles can be clearly seen in Fig. 8 (c); with the average velocity of the mixed material on the order of $15 \mathrm{~km} / \mathrm{s}$. From the diagnostics point of view, one of the important morphological features of the model is the dense material located at distances from the center of target greater than the original target radius. This material is a laterally expanding part of the target, chiefly composed of copper (see Fig. 8(a)) with the lateral velocities reaching $20 \mathrm{~km} / \mathrm{s}$ (Fig. 8 (d)).

\section{Discussion}

There are many parameters which can influence the results of the simulations. First one wants to determine the numerical resolution of the simulation. This is a balance between computational resources and the fidelity of the simulation. In addition, there are experimental variables such as the drive energy and small scale target variations which can influence the hydrodynamic evolution of the system.

In the case of the full-domain simulation considered in the previous section, we were limited to a resolution of around $\sim 1 \mu \mathrm{m}$ due to computational constraints. In Sec. 4.1 and 4.2, we consider small-domain simulations, where the domain covers only one half of the wavelength of the main perturbation on the $\mathrm{Cu} / \mathrm{CH}$ interface. These simulations are used to investigate the dependence on the numerical resolution and the impact of additional small-scale perturbations. For all of the smalldomain simulations presented here, the laser energy is $80 \mathrm{~kJ}$, and the intensity is approximately uniform over the whole computational domain. All simulations are done in Cartesian geometry and reflecting boundary conditions are applied at both the lower and the upper lateral $(x)$ boundary. The primary perturbation on the $\mathrm{Cu} / \mathrm{CH}$ interface has wavelength $\lambda_{1}=256 \mu \mathrm{m}$ and amplitude $a_{1}=24 \mu$, the domain size is $L_{z}=2560 \mu \mathrm{m}$ in the longitudinal direction and $L_{x}=128 \mu \mathrm{m}$ in the lateral direction. In Sec. 4.3 we use again full-domain simulations to investigate the dependence of the results on the laser drive energy. In Sec. 4.4 the Rayleigh-Taylor growth observed in the simulations is compared with the predictions from a buoyancy-drag model.

\subsection{Resolution study}

We first perform a series of small-domain simulations at successively higher resolutions (the series SL* in Table 1). The aim of this study is to determine at which spatial scale the smallscale structures no longer affect the overall morphology of the flow. Fig. 9 shows density plots for this simulation series, starting from 32 grid cell per wavelength effective resolution (simulation SL32) and successively doubling the resolution up to 2048 cells per wavelength (simulation SL2048). As one can see from these plots, the shock position is converged at a resolution of 64 cells per wavelength. With increasing resolution,
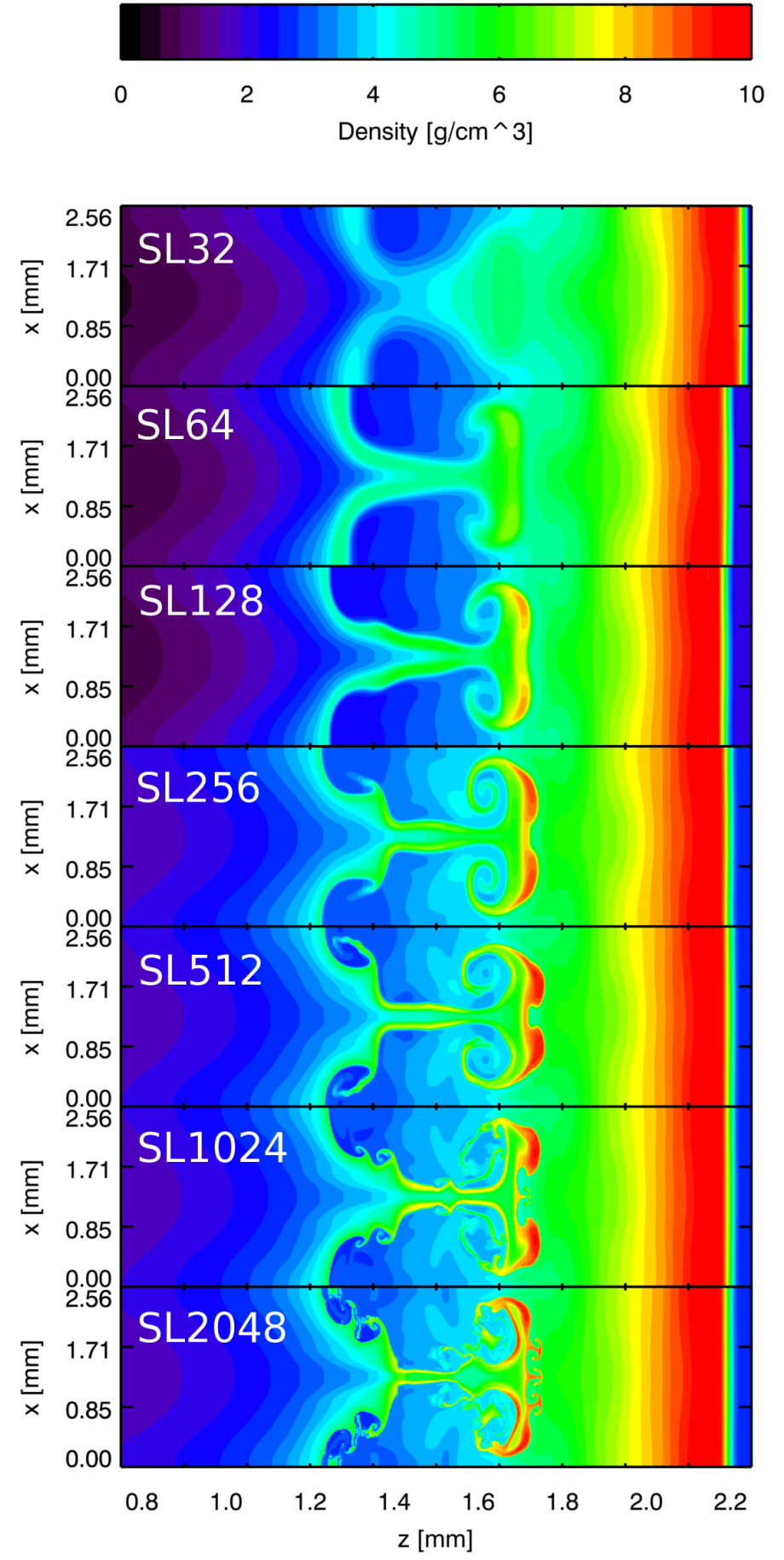

Figure 9: Resolution study. Plots of the density for simulations that have a single-mode perturbation on the $\mathrm{Cu} / \mathrm{CH}$ interface, with wavelength $\lambda_{1}=$ $256 \mu \mathrm{m}$ and amplitude $a_{1}=24 \mu \mathrm{m}$, performed at different effective resolutions. The plots are taken at $t=200 \mathrm{~ns}$; at this time, the shock is located at $x \approx 2200 \mu \mathrm{m}$. 


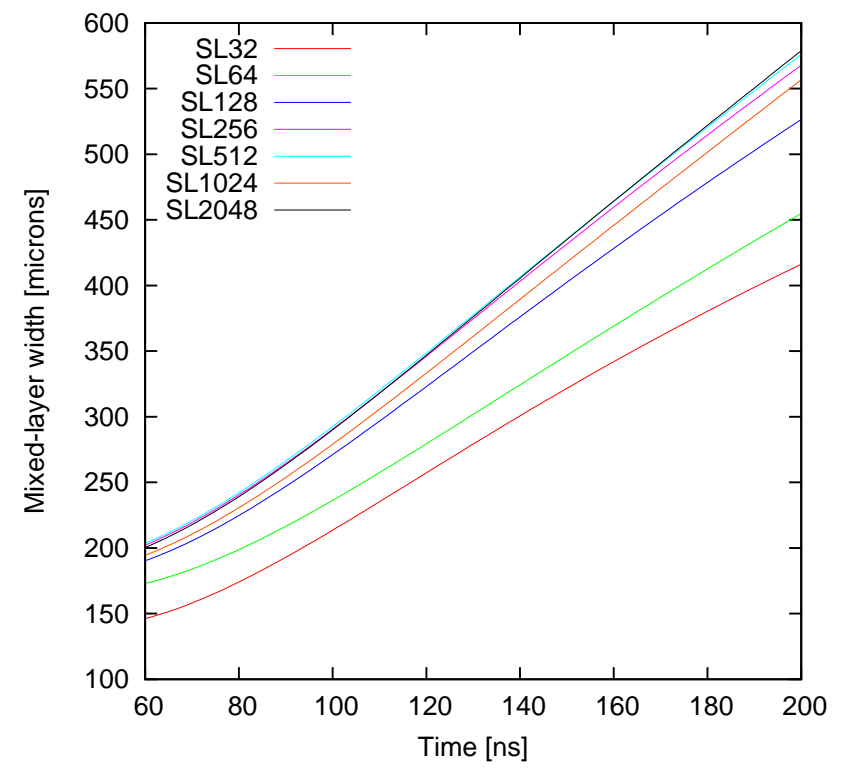

Figure 10: Plot of the mix layer width vs. time for the SL* simulation series. The mix layer width is defined as the region containing at least $1 \%$ of both copper and plastic.

the flow develops more and more small-scale structures. At the highest resolutions, secondary instabilites grow on top of the primary instability. The global morphology, however, appears to be basically converged at an effective of 256 cells per wavelength (simulation SL256).

This conclusion is further strengthened when looking at Fig. 10, where we plot the mix layer width for each of the simulations as a function of time. Here and in what follows, the mix layer width is defined as the width of the region containing at least $1 \%$ of both the heavy and the light fluid. The mix layer width is essentially converged for simulation SL256, changing only within a few percent in the higher resolution simulations. The mix layer width at the final time $(t=200 \mathrm{~ns})$ is $\approx 570 \mu \mathrm{m}$. Our results are in basic agreement with previous works concerning Rayleigh-Taylor growth in laser-driven shock experiments, which suggested that a numerical resolution of $\sim 10^{2}$ resolution elements per wavelength is sufficient to capture the general morphology of the flow and to determine basic integral quantities such as the mix layer width [3, 29].

\subsection{Sensitivity to fabrication perturbations}

In an actual experiment, the process of target fabrication will inevitably introduce small imperfections on component surfaces. Here, we consider the effect of additional, small-scale perturbations on the ablator surface, the ablator/copper interface and on the copper/plastic interface. While the manufacturing imperfections might be too small to be resolved in a fullscale numerical simulation, we can still use the small-domain simulations to provide bounds on the effect of such perturbations on the physics of interest.

We perform simulations with the same parameters as in the resolution study discussed in the previous subsection, except that we now add a secondary perturbation on the component

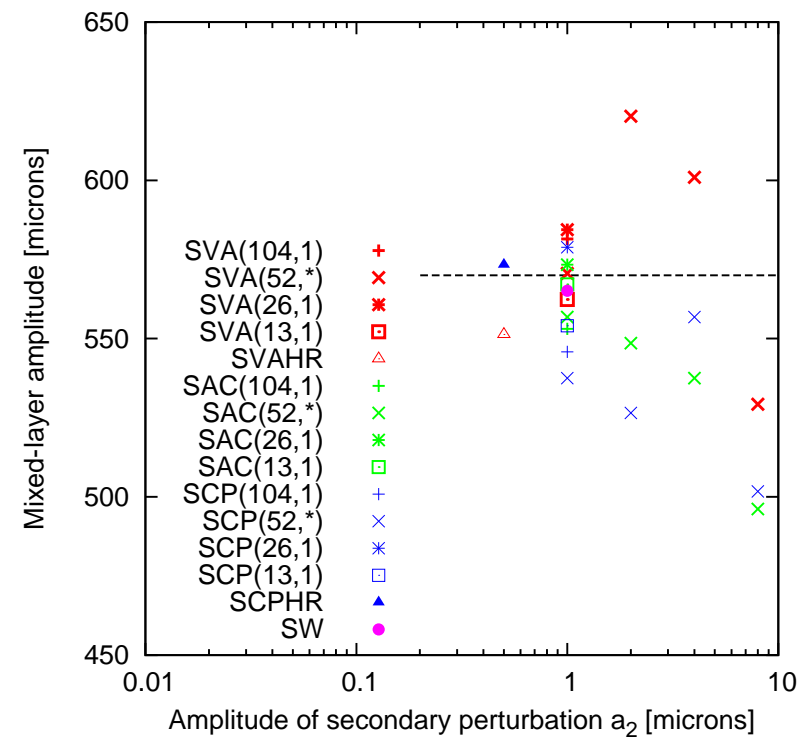

Figure 12: Mixed-layer amplitude at $t=200 \mathrm{~ns}$ in simulations with a smallscale secondary perturbation. For the meaning of the simulation labels, see Table 1. The horizontal line is the converged mix layer width of $570 \mu \mathrm{m}$ found in the resolution study.

interfaces. We first perform a series of simulations where a secondary perturbation with wavelength $\lambda_{2}$ and amplitude $a_{2}$ is imposed on either the ablator surface (SVA $\left(\lambda_{2}, a_{2}\right)$ series), the ablator/copper interface $\left(\operatorname{SAC}\left(\lambda_{2}, a_{2}\right)\right.$ series) or the copper/plastic interface ( $\operatorname{SCP}\left(\lambda_{2}, a_{2}\right)$ series). For each case, we perform four simulations with fixed wavelength $\lambda_{2}=52 \mu \mathrm{m}$, and the amplitude $a_{2}$ varying from $8 \mu \mathrm{m}$ down to $1 \mu \mathrm{m}$, as well as three more simulations where the amplitude is kept fixed at $a_{2}=1 \mu \mathrm{m}$ and the wavelength takes on the values $\lambda_{2}=13,26$ and $104 \mu \mathrm{m}$. All simulations are carried out at an effective numerical resolution of 1024 cells per primary perturbation wavelength.

Fig. 11 shows density plots for the simulations with fixed secondary perturbations wavelength. For all three cases, the overall morphology of the spike is similar for secondary perturbation amplitudes $\leq 1 \mu \mathrm{m}$. Only at larger perturbation amplitudes is the morphology of the spike significantly affected.

In addition to the simulations already mentioned, we perform two simulations (SVAHR and SCPHR), which have a secondary perturbation with wavelength $\lambda_{2}=52 \mu$ and amplitude $a_{2}=0.5 \mu \mathrm{m}$ on the ablator surface or on the copper/plastic interface, respectively. These two simulations are performed at an effective resolution of 2048 grid cells per primary perturbation wavelength, in order to ensure that the secondary perturbation is adequately resolved. We also perform a simulation (labeled 'SW'), which has secondary perturbations with the same wavelength $\lambda_{2}=52 \mu \mathrm{m}$ and amplitude $a_{2}=1 \mu \mathrm{m}$ on all three interfaces simultaneously. Fig. 12 shows the final $(t=200 \mathrm{~ns}) \mathrm{mix}$ layer amplitudes obtained for all simulations. For amplitudes greater than $2 \mu \mathrm{m}$, the mix layer amplitude varies significantly with the amplitude of the secondary perturbation. The mix layer amplitude does no longer depend on the amplitude of the secondary perturbation once $a_{2} \leq 1 \mu \mathrm{m}$, but is in a $\pm 5 \%$ range 

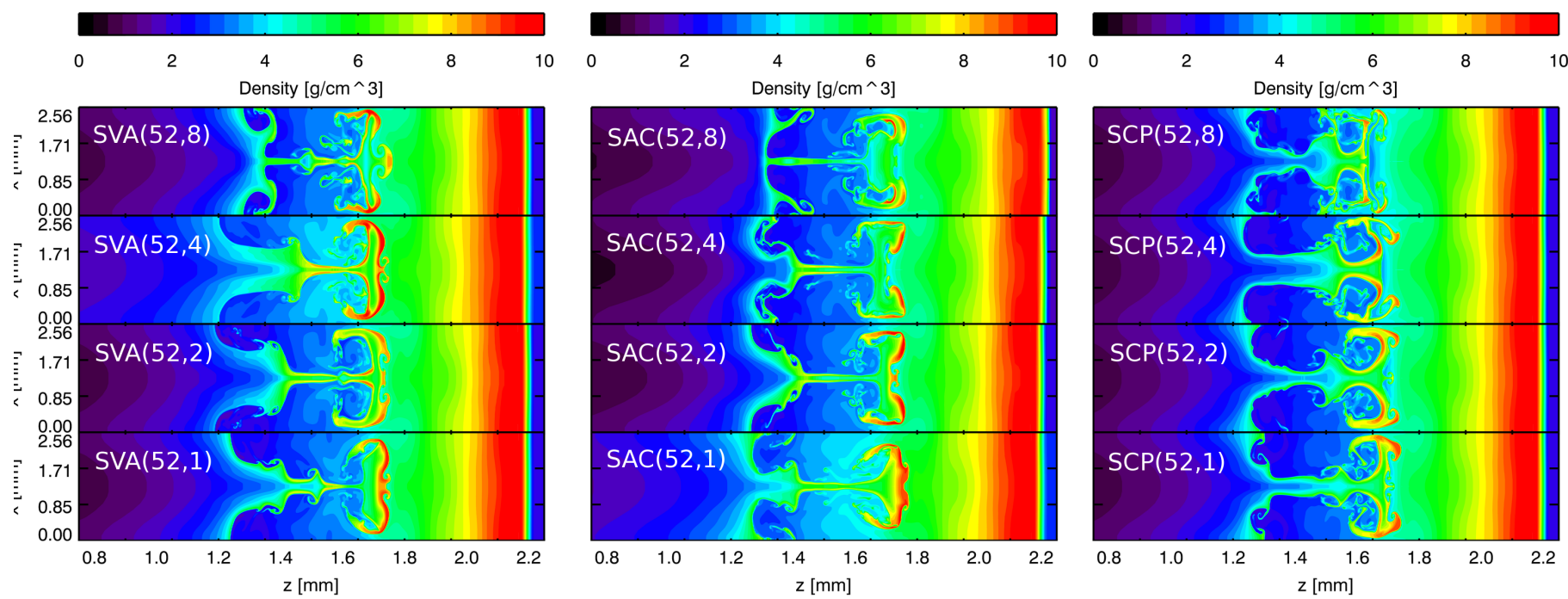

Figure 11: Shown are density plots taken at time $t=200 \mathrm{~ns}$ for small-domain simulations with a secondary perturbation with wavelength of $52 \mu \mathrm{m}$ on either the ablator surface (simulations labeled SVA), the ablator/copper interface (label SAC) or the copper/plastic interface (label SCP.) The amplitude of the secondary perturbation (the second number in brackets) varies from $8 \mu \mathrm{m}$ down to $0.5 \mu \mathrm{m}$.

from the $570 \mu \mathrm{m}$ value found in the resolution study.

Typical machining perturbations have amplitudes of less than 1 micron [2], and therefore we do not expect these perturbations to significantly alter the flow morphology in an experiment. The results also imply that the feed-through of perturbations on the ablation front resulting from RMI/RTI are not expected to significantly change the width of the mix layer, provided that their amplitude is $\lesssim 1 \mu \mathrm{m}$.

\subsection{Dependence on drive energy}

In order to determine the effect of the laser drive energy on the system evolution, we perform a series of simulations at different drive energies. Apart from the numerical resolution and the laser drive energy, the parameters of these simulations are identical to the parameters of the base model presented in Sec. 3. We consider the cases of $100 \mathrm{~kJ}, 200 \mathrm{~kJ}$ and $300 \mathrm{~kJ}$ drive energy (Fig. 13 (b)). The simulations are carried out at an effective resolution of $2 \mu \mathrm{m}$. In Fig. 13 density plots as well as simulated radiographs are shown for each case. The plots are made at times where the shock front is at $z=2000 \mu \mathrm{m}$, i.e. the dimensionless time is similar for all plots.

As seen in the density plots (Fig. 13, plots (a), (c) and (d)), the morphology of the system does not vary dramatically between the different cases. The mix layer width increases and the spikes become thinner with higher drive energy. Concerning the synthetic radiographs, (plots (b), (d) and (f) of Fig. 13), the contrast in the mixing region improves significantly when going to higher energy. Also, the shock front becomes narrower and easier to identify. Based on these simulation results, we thus expect to be able to better interpret the experimental radiographs at higher laser energies.

\subsection{Comparison with buoyancy-drag model}

As long as the amplitude-to-wavelength ratio $h / \lambda$ is smaller than about 0.1 , a single-mode perturbation on a RayleighTaylor instable interface will grow exponentially in time, i.e.

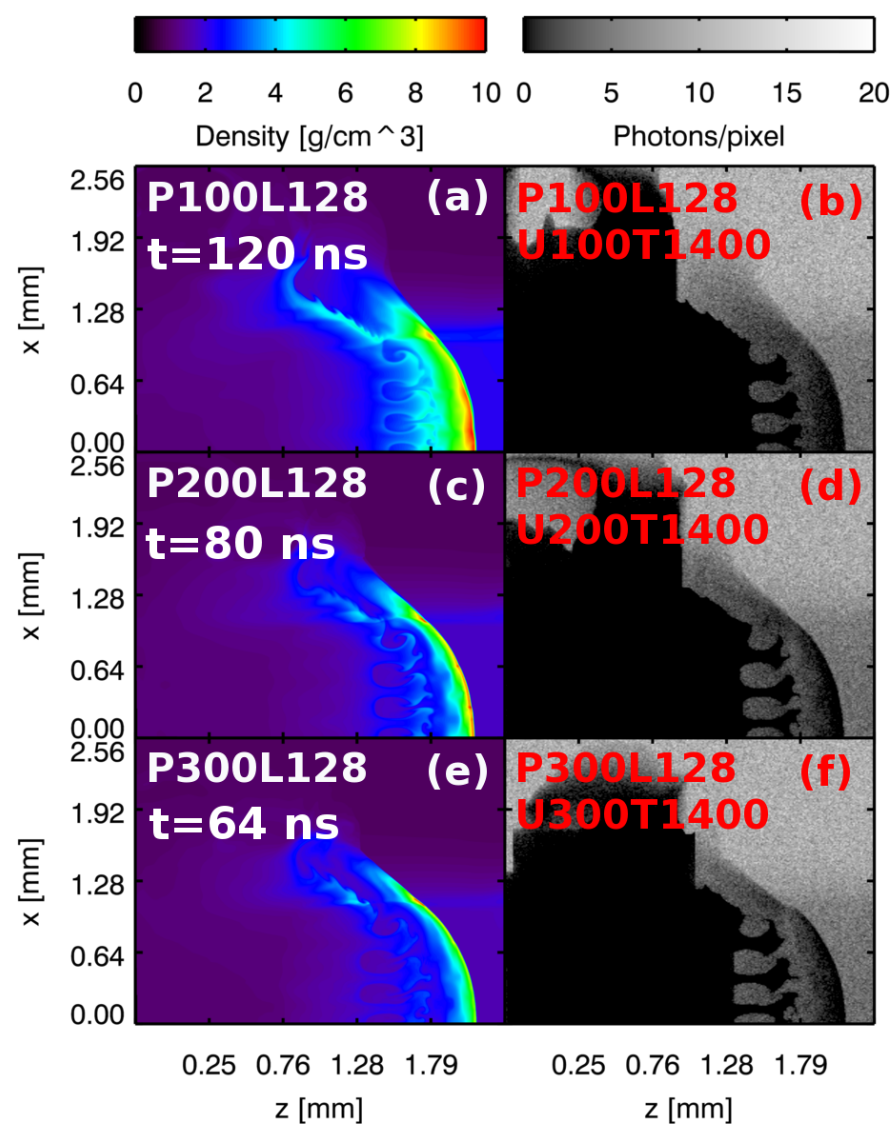

Figure 13: Density plots and synthetic radiographs for simulations covering the full experimental domain done at different drive energies. The laser drive energies are (from top to bottom): $100 \mathrm{~kJ}, 200 \mathrm{~kJ}$ and $300 \mathrm{~kJ}$. The plots have been made at times $t=120 \mathrm{~ns}, 80 \mathrm{~ns}$ and $64 \mathrm{~ns}$, respectively. For the calculation of each of the radiographs, data from a perturbed Cartesian $(\mathrm{P} * \mathrm{~L} 128)$ simulation has been combined with data from the corresponding unperturbed cylindrical (U*T1400 simulation, as described in Sec. 2.4. 
$h(t) \propto \mathrm{e}^{\gamma t}$. During that linear phase of instability growth, the growth rate is given by $\gamma=\sqrt{A k g}$, with $A$ the Atwood number, $k=2 \pi / \lambda$ the mode number and $g$ the interface acceleration.

During the subsequent non-linear phase of the evolution, $h / \lambda \gg 0.1$ and the mix layer width can be described using buoyancy-drag models [25, 4, 31, and references therein]. In order to assess how well our design captures the behaviour of the Rayleigh-Taylor instability at large scales, we compare the evolution of the mix layer width in our simulations with predictions of a particular buoyancy-drag model. Since in our case the target expansion contributes significantly to the growth of the mix layer, it has to be included in the calculation. The mix layer amplitude (defined as one half of the mix layer width) can then be calculated as [28]

$$
\frac{\mathrm{d} h}{\mathrm{~d} t}=\frac{\mathrm{d} h_{\mathrm{RTI}}}{\mathrm{d} t}+\frac{\mathrm{d} h_{\exp }}{\mathrm{d} t},
$$

where $\mathrm{d} h_{\mathrm{RTI}} / \mathrm{d} t$ is the contribution due to Rayleigh-Taylor instability, and $\mathrm{d} h_{\exp } / \mathrm{d} t$ is the contribution due to target expansion. Following Oron et al. (2001) and Dimonte (2000), the mix layer amplitude in the buoyancy-drag model is given by

$$
\left(\rho_{1}+C_{a} \rho_{2}\right) \frac{\mathrm{d}^{2} h_{\mathrm{RTI}}}{\mathrm{d} t^{2}}=\left(\rho_{2}-\rho_{1}\right) g-\frac{C_{d}}{L} \rho_{2}\left(\frac{\mathrm{d} h_{\mathrm{RTI}}}{\mathrm{d} t}\right)^{2} .
$$

In this equation, $\rho_{1}$ and $\rho_{2}$ are the limiting densities just to the left and to the right of the interface. In the case of twodimensional RTI that we are considering, $C_{a}=2$ and $C_{d}=6 \pi$ and the characteristic length scale $L$ is set equal to the mix layer amplitude $h$. The acceleration $g$ of the $\mathrm{Cu} / \mathrm{CH}$ interface $z_{\text {int }}$ is given by

$$
g=\frac{\mathrm{d}^{2} z_{\text {int }}}{\mathrm{d} t^{2}}
$$

The time-dependent quantities $\rho_{1}, \rho_{2}$ and $g$ are determined by averaging the data from a two-dimensional unperturbed simulation over the intervall $\left[0, R_{\text {pert }}\right]$ in the lateral direction. Plots of the interface acceleration for the three cases are shown in Fig. 14.

The contribution to the change in the mix layer amplitude coming from the target expansion is calculated as

$$
\frac{\mathrm{d} h_{\exp }}{\mathrm{d} t}=\frac{\Delta v_{z}}{2}
$$

where $\Delta v_{z}=v_{z}\left(z_{\text {int }}+h\right)-v_{z}\left(z_{\text {int }}-h\right)$ is the velocity gradient in the longitudinal direction.

Fig. 15 shows a comparison between the prediction of the mix layer width as predicted by the buoyancy-drag model and the result from the numerical simuations presented in Sec. 4.3. For all three cases considered, the match between the simulations and the buoyancy-drag model is excellent. The contribution to the mix layer growth from the target expansion is about $2 / 3$. For comparison, Kuranz et al. [22] found that in their experiment the target expansion contributed a factor of about $50 \%$ to the mix layer growth.

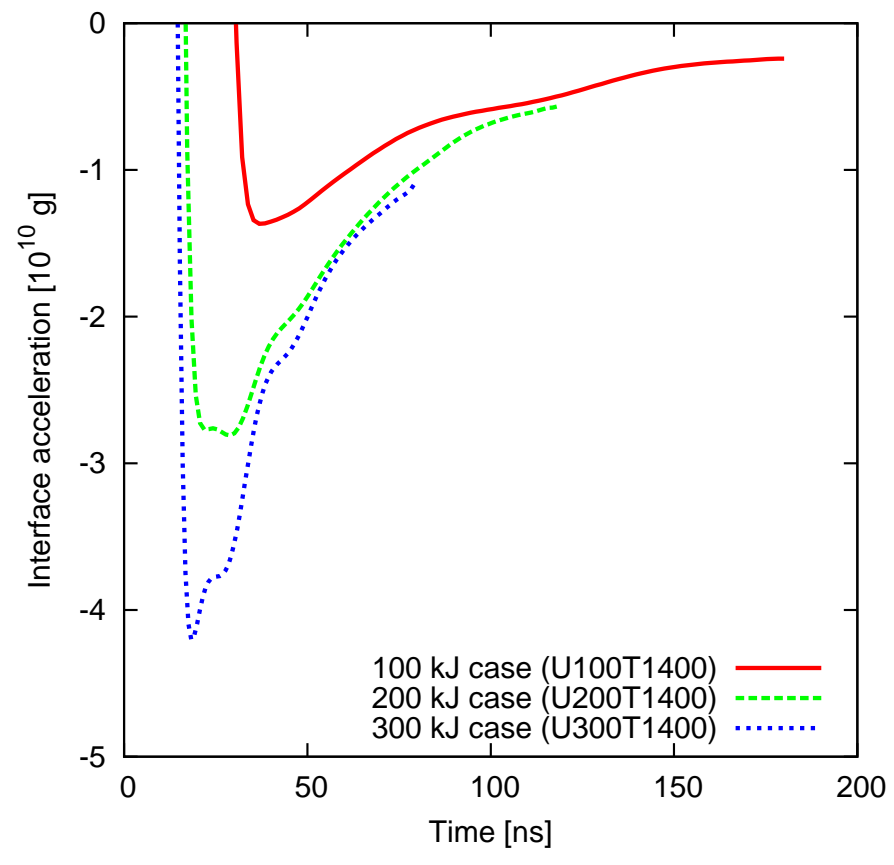

Figure 14: Interface acceleration measured in units of the earths surface gravity vs. time for unperturbed simulations at different drive energies. The acceleration is measured as the mean acceleration in the region $R<R_{\text {pert }}$.

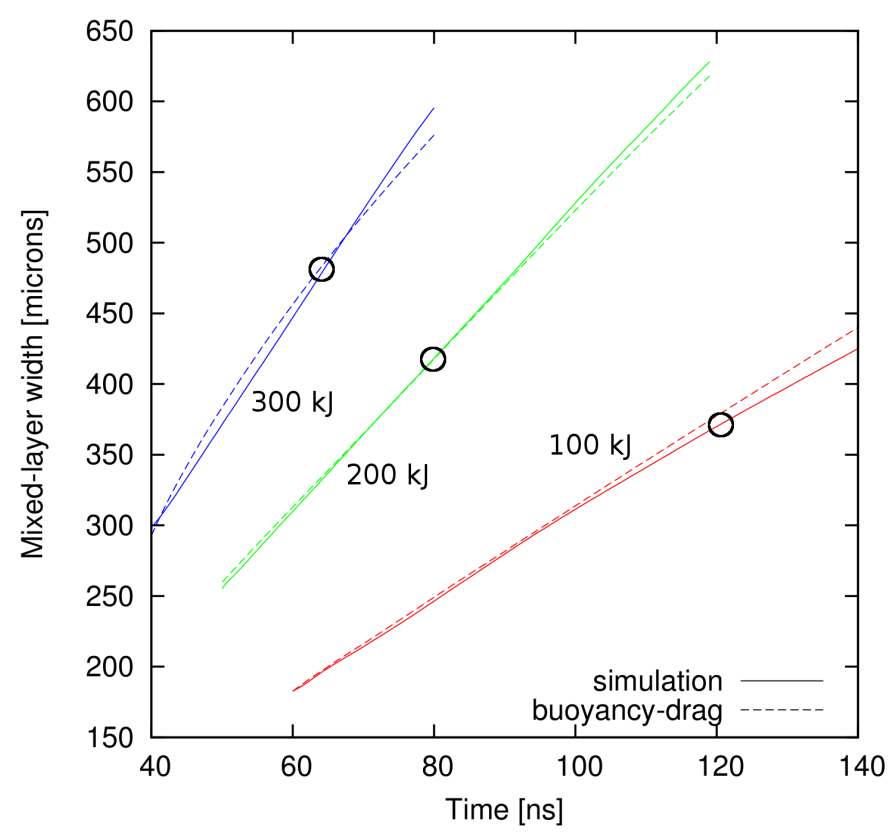

Figure 15: Comparison between the mix layer width as predicted by Eq. (3) (solid lines) and the result from the corresponding numerical simuations (dashed lines). The circles correspond to the times at which the plots in Fig. 13 were made. For the calculation of the mix layer width, we average the material fractions laterally from $R=0$ to $R=1400 \mu \mathrm{m}$ and define the mix layer width as the width of the region where the average material fraction is at least $1 \%$ for both the heavy and the light fluid. The starting times for the curves in this figure were chosen such that the interface position at this time is $\approx 1100 \mu \mathrm{m}$ for all three cases. Note that the curves contain the contribution to the mix layer width from both the target expansion and the Rayleigh-Taylor growth. 


\section{Conclusions}

In this paper, we have presented design simulations of a directly-driven Rayleigh-Taylor experiment for the National Ignition Facility, with relevance to material mixing in supernovae. Performing such an experiment on NIF would not only be helpful in developing the direct-drive experimental platform on NIF but would also serve as a stepping stone for more sophisticated experiments studying the Rayleigh-Taylor instability in spherical geometry, which would be a much more realistic model for the material mixing occuring in supernovae.

We have described in detail the numerical setup for the design simulations that were done using the adaptive-mesh radiation hydrodynamics code CRASH. Assuming that the experiment will be diagnosed via $x$-ray radiography, we optimized the target size and the laser drive configuration such as to achieve optimal contrast in the radiographs; and we demonstrated that the experiment can be properly diagnosed with the diagnostic capabilities already present on NIF.

We have discussed in some detail the results of one particular high-resolution simulation which covered the whole experimental domain. In addition, we performed small-domain simulations, where only one half of a spike was simulated and where we were able to achieve effective resolutions as high as 2048 cells per perturbation wavelength. By performing simulations at successively higher resolutions, it was found that the global morphology of the flow is converged at a resolution of 256 cells per wavelength. Motivated by the fact that the process of target manufacturing inevitably introduces small imperfections on component surfaces, we also explored the impact of small-scale secondary perturbations, finding that the flow morphology is significantly affected only for perturbations with an amplitude higher than $1 \mu \mathrm{m}$, which is well well above the typical length scale of target imperfections.

Finally, we looked at how the outcome changes when varying the drive energy, considering energies in the range of 100$300 \mathrm{~kJ}$. While the overall morphology did not change drastically (when viewed at the same characteristic time), the variations in the mix layer was greater for the higher energy models and the radiographic contrast was also found to improve with increasing drive energy. Comparison of the mix layer with found in the simulations with the predictions of a buoyancy-drag model showed excellent agreement. The target expansion was found to contribute about $2 / 3$ to the mix layer width, which is higher than in previous experiments of this type, which were performed at lower drive energy.

\section{Acknowledgments}

We thank two anonymous reviewers for providing thoughtful comments that helped to improve the manuscript. MF and TP were supported by the DOE grant DE-FG52-09NA29548 and the NSF grant AST-1109113. This research used resources of the National Energy Research Scientific Computing Center, which is supported by the Office of Science of the U.S. Department of Energy under Contract No. DE-AC02-05CH11231.

\section{Appendix A. Glossary of acronyms and notation}

$\begin{array}{ll}\text { NIF } & \text { National Ignition Facility } \\ \text { RMI } & \text { Richtmeyer-Meshkov instability } \\ \text { RTI } & \text { Rayleigh-Taylor instability } \\ \text { SN } & \text { supernova } \\ \text { SNR } & \text { signal-to-noise-ratio } \\ R_{\text {tube }} & \text { target radius (see Fig. } 1) \\ R_{\text {pert }} & \text { "perturbation radius" (see Fig. 1) } \\ R_{\text {cart }} & \text { radius below which Cartesian data is used (Sec. 2.4) } \\ R_{\text {wash }} & \text { laser cut-off radius (see Fig. 1) } \\ R_{\text {off }} & 50^{\circ} \text { beams offset (Sec. 2.3) } \\ P_{\text {ctr }} & \left.\text { laser power in the } 23^{\circ} \text { and } 30^{\circ} \text { beams (Sec. } 2.3\right) \\ P & \text { total laser power } \\ E & \text { total laser energy, } E \approx P \cdot 3 \text { ns } \\ \lambda_{1} & \text { wavelength of primary perturbation (see Fig. 1) } \\ a_{1} & \text { amplitude of primary perturbation (see Fig. 1) } \\ \lambda_{2} & \text { wavelength of secondary perturbation (Sec. } 4.2) \\ a_{2} & \text { amplitude of secondary perturbation (Sec. } 4.2)\end{array}$

\section{References}

[1] D. Arnett, B. Fryxell, and E. Mueller. Instabilities and nonradial motion in SN 1987A. Astrophys. J., 341:L63-L66, June 1989.

[2] M. Basko, T. Löwer, V. N. Kondrashov, A. Kendl, R. Sigel, and J. MeyerTer-Vehn. Optical probing of laser-induced indirectly driven shock waves in aluminum. Phys. Rev. E, 56:1019-1031, July 1997.

[3] A. C. Calder, B. Fryxell, T. Plewa, R. Rosner, L. J. Dursi, V. G. Weirs, T. Dupont, H. F. Robey, J. O. Kane, B. A. Remington, R. P. Drake, G. Dimonte, M. Zingale, F. X. Timmes, K. Olson, P. Ricker, P. MacNeice, and H. M. Tufo. On Validating an Astrophysical Simulation Code. Astrophys. J. Suppl. Ser., 143:201-229, November 2002.

[4] G. Dimonte. Spanwise homogeneous buoyancy-drag model for RayleighTaylor mixing and experimental evaluation. Physics of Plasmas, 7:22552269, June 2000.

[5] R. P. Drake, J. J. Carroll, III, K. Estabrook, S. G. Glendinning, B. A. Remington, R. Wallace, and R. McCray. Development of a Laboratory Environment to Test Models of Supernova Remnant Formation. Astrophys. J., 500:L157, June 1998.

[6] R. P. Drake, H. F. Robey, O. A. Hurricane, Y. Zhang, B. A. Remington, J. Knauer, J. Glimm, D. Arnett, J. O. Kane, K. S. Budil, and J. Grove. Experiments to Produce a Hydrodynamically Unstable, Spherically Diverging System of Relevance to Instabilities in Supernovae. Astrophys. J., 564:896-908, January 2002.

[7] National Ignition Facility. User guide. https://lasers.1lnl.gov/ for_users/pdfs/2012user_guide.pdf, 2012.

[8] D. H. Froula, T. J. Kessler, I. V. Igumenshchev, R. Betti, V. N. Goncharov, H. Huang, S. X. Hu, E. Hill, J. H. Kelly, D. D. Meyerhofer, A. Shvydky, and J. D. Zuegel. Mitigation of cross-beam energy transfer: Implication of two-state focal zooming on OMEGA. Physics of Plasmas, 20(8):082704, August 2013.

[9] B. Fryxell, D. Arnett, and E. Mueller. Instabilities and clumping in SN 1987A. I - Early evolution in two dimensions. Astrophys. J., 367:619634, February 1991.

[10] A. Gawryszczak, J. Guzman, T. Plewa, and K. Kifonidis. Non-spherical core collapse supernovae. III. Evolution towards homology and dependence on the numerical resolution. Astron. Astrophys., 521:A38, October 2010.

[11] V. N. Goncharov. Analytical Model of Nonlinear, Single-Mode, Classical Rayleigh-Taylor Instability at Arbitrary Atwood Numbers. Physical Review Letters, 88(13):134502, April 2002.

[12] M. J. Grosskopf, R. P. Drake, C. C. Kuranz, A. R. Miles, J. F. Hansen, T. Plewa, N. Hearn, D. Arnett, and J. C. Wheeler. Modeling of multiinterface, diverging, hydrodynamic experiments for the National Ignition Facility. Astrophys. Space Sci., 322:57-63, August 2009. 
[13] M.J. Grosskopf, R.P. Drake, A.R. Miles, T. Plewa, and C.C. Kuranz. Modeling of aspheric, diverging hydrodynamic instability experiments on the national ignition facility. High Energy Density Physics, 9(3):439 - 447, 2013.

[14] N. J. Hammer, H.-T. Janka, and E. Müller. Three-dimensional Simulations of Mixing Instabilities in Supernova Explosions. Astrophys. J., 714:1371-1385, May 2010.

[15] J. Kane, D. Arnett, B. A. Remington, S. G. Glendinning, G. Bazn, E. Mller, B. A. Fryxell, and R. Teyssier. Two-dimensional versus threedimensional supernova hydrodynamic instability growth. The Astrophysical Journal, 528(2):989, 2000.

[16] J. Kane, D. Arnett, B. A. Remington, S. G. Glendinning, J. Castor, R. Wallace, A. Rubenchik, and B. A. Fryxell. Supernova-relevant hydrodynamic instability experiments on the nova laser. The Astrophysical Journal Letters, 478(2):L75, 1997.

[17] K. Kifonidis, T. Plewa, H.-T. Janka, and E. Müller. Nucleosynthesis and Clump Formation in a Core-Collapse Supernova. Astrophys. J., 531:L123-L126, March 2000

[18] K. Kifonidis, T. Plewa, H.-T. Janka, and E. Müller. Non-spherical core collapse supernovae. I. Neutrino-driven convection, Rayleigh-Taylor instabilities, and the formation and propagation of metal clumps. Astron. Astrophys., 408:621-649, September 2003.

[19] K. Kifonidis, T. Plewa, L. Scheck, H.-T. Janka, and E. Müller. Nonspherical core collapse supernovae. II. The late-time evolution of globally anisotropic neutrino-driven explosions and their implications for SN 1987 A. Astron. Astrophys., 453:661-678, July 2006.

[20] C. C. Kuranz, R. P. Drake, M. J. Grosskopf, A. Budde, C. Krauland, D. C. Marion, A. J. Visco, J. R. Ditmar, H. F. Robey, B. A. Remington, A. R. Miles, A. B. R. Cooper, C. Sorce, T. Plewa, N. C. Hearn, K. L. Killebrew, J. P. Knauer, D. Arnett, and T. Donajkowski. Threedimensional blast-wave-driven Rayleigh-Taylor instability and the effects of long-wavelength modes. Physics of Plasmas, 16(5):056310, May 2009.

[21] C. C. Kuranz, R. P. Drake, M. J. Grosskopf, B. Fryxell, A. Budde, J. F Hansen, A. R. Miles, T. Plewa, N. Hearn, and J. Knauer. Spike morphology in blast-wave-driven instability experiments. Physics of Plasmas, 17(5):052709, May 2010

[22] C. C. Kuranz, R. P. Drake, E. C. Harding, M. J. Grosskopf, H. F. Robey, B. A. Remington, M. J. Edwards, A. R. Miles, T. S. Perry, B. E. Blue T. Plewa, N. C. Hearn, J. P. Knauer, D. Arnett, and D. R. Leibrandt. TwoDimensional Blast-Wave-Driven Rayleigh-Taylor Instability: Experiment and Simulation. Astrophys. J., 696:749-759, May 2009.

[23] C. C. Kuranz, R. P. Drake, D. R. Leibrandt, E. C. Harding, H. F. Robey, A. R. Miles, B. E. Blue, J. F. Hansen, H. Louis, M. Bono, J. Knauer, D. Arnett, and C. A. Meakin. Progress Toward the Study of Laboratory Scale, Astrophysically Relevant, Turbulent Plasmas. Astrophys. Space Sci., 298:9-16, July 2005.

[24] O.L. Landen, D. R. Farley, S. G. Glendinning, L. M. Logory, P.M. Bell, J.A. Koch, F. D. Lee, D.K. Bradley, D.H. Kalantar, C.A. Back, and R.E. Turner. X-ray backlighting for the national ignition facility (invited). $R e$ view of Scientific Instruments, 72(1):627-634, 2001.

[25] D. Layzer. On the Instability of Superposed Fluids in a Gravitational Field. Astrophys. J., 122:1, July 1955.

[26] G. Malamud, M.J. Grosskopf, and R.P. Drake. Conceptual design of a rayleightaylor experiment to study bubble merger in two dimensions on \{NIF\}. High Energy Density Physics, 11(0):17 - 25, 2014.

[27] E.E. Meshkov. Instability of the interface of two gases accelerated by a shock wave. Fluid Dynamics, 4(5):101-104, 1969.

[28] A. R. Miles. The Blast-Wave-Driven Instability as a Vehicle for Understanding Supernova Explosion Structure. Astrophys. J., 696:498-514, May 2009.

[29] A. R. Miles, D. G. Braun, M. J. Edwards, H. F. Robey, R. P. Drake, and D. R. Leibrandt. Numerical simulation of supernova-relevant laser-driven hydro experiments on OMEGA. Physics of Plasmas, 11:3631-3645, July 2004.

[30] Frank Modica, Tomasz Plewa, and Andrey Zhiglo. The braginskii model of the rayleigh-taylor instability. i. effects of self-generated magnetic fields and thermal conduction in two dimensions. High Energy Density Physics, 9(4):767 - 780, 2013

[31] D. Oron, L. Arazi, D. Kartoon, A. Rikanati, U. Alon, and D. Shvarts Dimensionality dependence of the Rayleigh-Taylor and RichtmyerMeshkov instability late-time scaling laws. Physics of Plasmas, 8:2883-
2889, June 2001.

[32] J.W. Strutt (Lord Rayleigh). Sci. Pap., 2:200, 1900.

[33] R.D. Richtmeyer. Taylor instability in shock acceleration of compressible fluids. Comm. Pure Appl. Math., 13:297-319, 1960.

[34] H. F. Robey, Y. Zhou, A. C. Buckingham, P. Keiter, B. A. Remington, and R. P. Drake. The time scale for the transition to turbulence in a high Reynolds number, accelerated flow. Physics of Plasmas, 10:614-622, March 2003.

[35] D. Ryutov, R. P. Drake, J. Kane, E. Liang, B. A. Remington, and W. M. Wood-Vasey. Similarity Criteria for the Laboratory Simulation of Supernova Hydrodynamics. Astrophys. J., 518:821-832, June 1999.

[36] G. Taylor. Proc. R. Soc. London A, 201:192, 1950.

[37] B. van der Holst, G. Tóth, I. V. Sokolov, K. G. Powell, J. P. Holloway, E. S. Myra, Q. Stout, M. L. Adams, J. E. Morel, S. Karni, B. Fryxell, and R. P. Drake. CRASH: A Block-adaptive-mesh Code for Radiative Shock Hydrodynamics - Implementation and Verification. Astrophys. J. Suppl. Ser., 194:23, June 2011.

[38] B. van der Holst, G. Tóth, I. V. Sokolov, B. R. Torralva, K. G. Powell, R. P. Drake, M. Klapisch, M. Busquet, B. Fryxell, and E. S. Myra. Simulating radiative shocks with the CRASH laser package. High Energy Density Physics, 9:8-16, March 2013. 